\title{
Dynamically and Quasiforbidden Transitions in Photoionization of Open-Shell Atoms: A Combined Experimental and Theoretical Study
}

\author{
D. Cubaynes, ${ }^{1,2}$ M. Meyer, ${ }^{2}$ A. N. Grum-Grzhimailo, ${ }^{3}$ J.-M. Bizau, ${ }^{1,2}$ E. T. Kennedy, ${ }^{4}$ J. Bozek, ${ }^{5}$ M. Martins, ${ }^{6}$ \\ S. Canton, ${ }^{5,7}$ B. Rude, ${ }^{5}$ N. Berrah, ${ }^{7}$ and F. J. Wuilleumier ${ }^{1,2}$ \\ ${ }^{1}$ LIXAM, UMR CNRS 8624, University Paris Sud, Bâtiment 350, 91405 Orsay Cedex, France \\ ${ }^{2}$ LURE, Centre Universitaire Paris-Sud, Bâtiment 209D, 91898 Orsay Cedex, France \\ ${ }^{3}$ Institute of Nuclear Physics, Moscow State University, Moscow 119992, Russia \\ ${ }^{4}$ School of Physical Sciences/NCPST, Dublin City University, Glasnevin, Dublin 9, Ireland \\ ${ }^{5}$ Advanced Light Source, Lawrence Berkeley National Laboratory, Berkeley, California 94720, USA \\ ${ }^{6}$ Institut für Experimentalphysik, Universität Hamburg, D-22761 Hamburg, Germany \\ ${ }^{7}$ Physics Department, Western Michigan University, Kalamazoo, Michigan 49008, USA
}

(Received 19 December 2003; published 11 June 2004)

Completely fine-structure-resolved photoelectron spectra produced from sodium atoms selectively excited into the $\mathrm{Na}^{*} 2 p^{6} 3 p^{2} P_{1 / 2}$ and ${ }^{2} P_{3 / 2}$ states were obtained using a third generation synchrotron source in conjunction with laser pumping and high-resolution spectrometry. The spectra show dramatically different behaviors. The strong variations observed in the regions of the $\mathrm{Na}^{+}$ $2 p^{5} 3 p^{1,3} L_{J}$ photolines and the $\mathrm{Na}^{+} 2 p^{5} 4 p^{1,3} L_{J}$ shakeup satellites are explained within a generalized geometrical model, accounting for the intermediate angular momentum coupling in the ionic states.

DOI: 10.1103/PhysRevLett.92.233002

PACS numbers: $32.80 . \mathrm{Hd}, 32.80 . \mathrm{Fb}$

The interaction of ionizing radiation with atoms is a key fundamental process in nature that occurs in many areas of physics. Probing atomic photoionization dynamics as clearly and rigorously as quantum mechanics permits provides an ideal testing ground for studying the complex interactions in the electronic cloud of an atom and enables critical comparison with predictions of the most advanced many-body theories. The interpretation of gas-phase studies offers an atomic perspective on the basic understanding of correlated systems that can be beneficial to other fields, such as plasmas, nanostructures, and highly correlated materials. In particular, photoelectron spectroscopy on open-shell atomic targets bring out specific phenomena arising from their nonspherical nature and developed fine structure [1-5].

In this Letter, we report clear evidence for the influence of the total angular momentum $J_{0}$ of the initial atomic state on photoionization transitions to final fine-structure ionic states. We have been able to control $J_{0}$ by tuning the wavelength of a dye laser to the energy of two different selected excited atomic states and to measure with a highresolution spectrometer the fine-structure resolved photoelectron spectra produced by innershell photoionization of these excited states. Independent of the photon energy, dramatic variations of the relative line intensities arising from single photoionization as well as from photoionization accompanied by excitation (shakeup satellites) were observed. These results represent a clear example of quasiforbidden transitions in open-shell atomic systems as introduced ten years ago by Pan and Starace [6] going beyond the geometrical model $[1,7,8]$. Violation of the geometrical relations was detected for $3 s$ photoionization of chlorine in the ground state at the photon energy of
$29.2 \mathrm{eV} \mathrm{[2],} \mathrm{in} \mathrm{good} \mathrm{agreement} \mathrm{with} \mathrm{the} \mathrm{predictions} \mathrm{of}$ Ref. [6]. Until now, no experiments have been carried out to study the quasiforbidden transitions in excited atoms. We find that, in addition to the clear observation of quasiforbidden transitions [6], other transitions can be either strongly depressed or enhanced. We interpret them by introducing the concept of dynamically forbidden transitions resulting from mutual cancellation of terms in the photoionization cross section. The dynamically forbidden transitions cannot be predicted by explicit selection rules based on conservation of angular momenta and parity. While studied for a particular atomic system, our results arise from general theoretical considerations, which are applicable to atomic and molecular systems in general.

Sodium was selected as a showcase for an open-shell atom because the fine structure of the final ionic state following innershell photoionization can be resolved almost completely and it offered an optimized wavelength range for the tuning of the laser. In the experiment, photons from the Advanced Light Source (ALS) synchrotron radiation facility at the Lawrence Berkeley National Laboratory photoionized the excited atoms in the $2 p$ subvalence shell, allowing us to observe, for the first time, clearly resolved strongly enhanced or depressed transitions, compared with the geometrical predicted values, and providing the opportunity to explore the physical reasons for this behavior for specific excited states of a fine-structure multiplet. The experimental difficulties for the corresponding experiments include the multiple demands of combining a good atomic beam with sufficient selectivity in populating the initial fine-structure atomic levels and detection at high-energy resolution of the 
resulting complex spectra of closely lying photoelectron lines $[2-4,9]$.

Vacuum ultraviolet radiation from the 10.0.1 undulator beam line was monochromatized and focused, together with the counterpropagating beam of a $\mathrm{cw}$ ring dye laser, into the source volume of the electron spectrometer. The wavelength of the laser was tuned either to the $2 p^{6} 3 s^{2} S_{1 / 2} \rightarrow 2 p^{6} 3 p^{2} P_{1 / 2}(\lambda=589.559 \mathrm{~nm})$ or to the $2 p^{6} 3 s^{2} S_{1 / 2} \rightarrow 2 p^{6} 3 p^{2} P_{3 / 2}(\lambda=589.995 \mathrm{~nm})$ transition. The relative density of atoms in the excited states was generally $10 \%$ to $20 \%$ for the case of ${ }^{2} P_{3 / 2}$ and of the order of $1 \%$ for the ${ }^{2} P_{1 / 2}$. The polarization of the synchrotron radiation (SR) beam was linear with a degree of polarization higher than 99\% [10]. The laser polarization was also linear and could be rotated around the direction of the laser beam. A resistively heated oven was used to generate a collimated sodium beam with an operating temperature between 300 and $360^{\circ} \mathrm{C}$. In these conditions, the density of sodium atoms in the ground state was varied over the $10^{12}-10^{13} \mathrm{~cm}^{-3}$ range. The electrons emitted from the sodium atoms were measured using a Scienta SES-200 hemispherical analyzer operated at the magic angle $\left(54^{\circ} 44^{\prime}\right)$ with respect to the SR polarization vector. The ultimate resolution reached in our experiment was $13 \mathrm{meV}$, as measured from the FWHM of isolated photolines.

The high brightness of the ALS beam combined with the very high-resolution electron spectrometer allowed the photoelectron spectrum to be measured with hitherto unprecedented precision. The final ionic states $2 p^{5} 3 p^{1,3} L_{J}$ were completely $L$ and $J$ resolvable with the single exception of the ${ }^{3} P_{0,1}$ (separated by only $1.5 \mathrm{meV}$ ). Thus, the relative intensity of every single ${ }^{1,3} L_{J}$ photoelectron line was measured, allowing sharp variations in individual electron lines to be observed. In previous photoionization experiments on $\mathrm{Na}$ atoms laser excited to the $2 p^{6} 3 p^{2} P_{3 / 2}$ state [11], a few broad groups of unresolved lines were recorded, allowing only configurationaveraged effects to be studied. Here we concentrate on the photolines due to single photoionization $\left(2 p^{5} 3 p^{1,3} L_{J}\right.$ final ionic states) as well as on the shakeup satellites $\left(2 p^{5} 4 p^{1,3} L_{J}\right.$ final ionic states) from the excited atom.

Figure 1 displays, in its two upper panels, details of the fine-structure resolved photoelectron spectra in the region of the $2 p^{5} 3 p^{1,3} L_{J}$ final ionic states resulting from both $2 p$ photoionization of the $2 p^{6} 3 p^{2} P_{3 / 2}$ [Fig. 1(a)] and $2 p^{6} 3 p^{2} P_{1 / 2}$ [Fig. 1(b)] laser-excited states. The relative intensities of the photolines do not show any photon energy dependence over the investigated energy range (45 to $120 \mathrm{eV}$ ). The ${ }^{1} S$ state, measured at lower kinetic energy, is not shown in the figure.

Since a state with angular momentum of $J_{0}=\frac{1}{2}$ cannot be aligned, the relative intensities of the photolines from the ${ }^{2} P_{1 / 2}$ are proportional to the cross sections to the $L S J$ final ionic states. When dealing with the ${ }^{2} P_{3 / 2}$ excited state, however, it is important to consider any possible

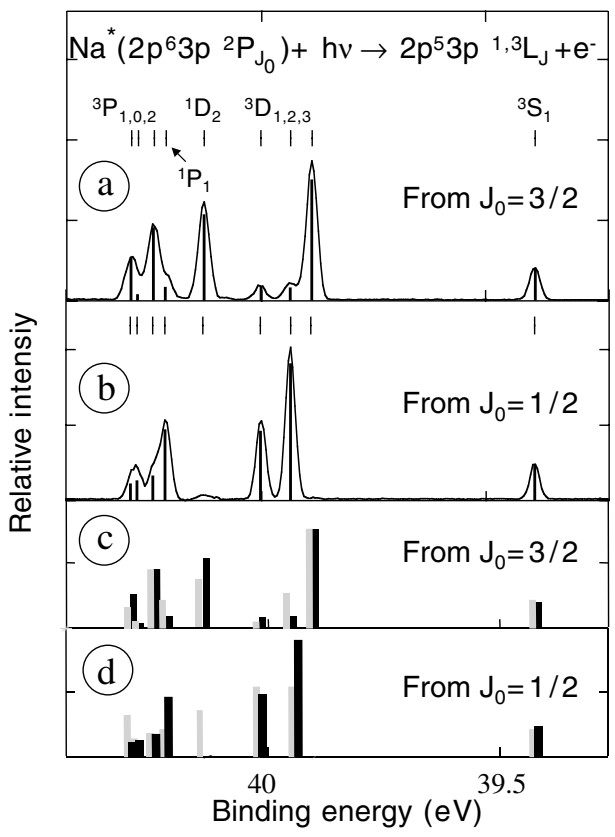

FIG. 1. Region of $\mathrm{Na}^{+} 2 p^{5} 3 p$ photoelectron spectra produced at $48 \mathrm{eV}$ by photoionization from laser-excited $\mathrm{Na}^{*}$ $2 p^{6} 3 p^{2} P_{3 / 2}$ (a) and ${ }^{2} P_{1 / 2}$ (b). (c),(d) Black bars show our theoretical results calculated in the generalized geometrical model; grey bars present results of the geometrical model. The experimental spectra are normalized to the intensity of the $2 p^{5} 3 p^{3} S_{1}$ final ionic state calculated in the generalized geometrical model.

alignment effects. With the laser tuned to the ${ }^{2} P_{3 / 2}$ transition, we observed variations of the relative intensities of some of the photolines, especially for the ${ }^{1,3} S$ state, when varying the angle between both polarization axes, indicating that the excited atoms were partly aligned, in spite of the very high sodium atom density of $10^{13} \mathrm{~cm}^{-3}$. In previous experiments on polarization effects in pumpprobe experiments [12], it was determined, by fluorescence analysis, that alignment effects continuously decreased with increasing vapor densities from about $2 \times$ $10^{10} \mathrm{~cm}^{-3}$ and vanished at about $10^{12} \mathrm{~cm}^{-3}$. In the present case, however, alignment effects are still present at even higher beam densities, because with a third generation SR source the focal spot of the photon beam is very small (less than $0.1 \mathrm{~mm}^{2}$ ). On this spatial scale, depolarization by radiation trapping and collisions is drastically reduced. To minimize the influence of the alignment on the relative intensities of the photoelectron lines displayed in Fig. 1, the sum of spectra taken at parallel and perpendicular polarizations of the laser and SR beams was used. This results in inaccuracy in the relative intensities due to the alignment not exceeding $15 \%$, which does not change any of the conclusions deduced from our measurements.

The observed spectra are dramatically different for ionization from the initial states ${ }^{2} P_{3 / 2}$ and ${ }^{2} P_{1 / 2}$ (upper two panels in Fig. 1). With ${ }^{2} P_{3 / 2}$ optical excitation, the photoionization cross section associated with the ${ }^{3} D_{3}$ 
final ionic state is the strongest, while the two other components of the multiplet, ${ }^{3} D_{1}$ and ${ }^{3} D_{2}$, correspond to the weakest oscillator strength of the spectrum. The cross section to the ${ }^{1} D_{2}$ ionic state is almost equal to the cross section to the ${ }^{3} D_{3}$ state. Photoionization of the ${ }^{2} P_{1 / 2}$ state reveals a profound redistribution of the oscillator strength. The cross sections to the ${ }^{3} D_{3}$ and ${ }^{1} D_{2}$ final ionic states have completely vanished or are drastically reduced. The cross sections to the two other components of the multiplet, ${ }^{3} D_{1}$ and ${ }^{3} D_{2}$, now dominate the spectrum. Similar effects are also observed for the ${ }^{1,3} P$ components, with a transfer of the oscillator strength from the ${ }^{3} P_{2}$ final ionic state in the case of photoionization from the ${ }^{2} P_{3 / 2}$ excited state, to the ${ }^{1} P_{1}$ state for ionization from the ${ }^{2} P_{1 / 2}$ state.

Figure 1 also displays, in the two upper panels, as vertical lines superimposed on the experimental data, the results of Cowan code calculations carried out in the single-configuration Hartree-Fock (HF) approximation [13]. The calculations accurately reproduce the experimental data in terms of relative cross sections and variations between the ${ }^{2} P_{1 / 2}$ and ${ }^{2} P_{3 / 2}$ cases. In order to explore the underlying physics determining the differences in behavior in the two cases, we have carried out a detailed theoretical analysis of photoionization from the $\mathrm{Na}^{*}$ laser-excited states. The analysis is done for our experimental conditions and aims at finding the cross sections from the observed line intensities, determining any alignment of the laser-excited state from the line intensities, and extracting maximum information on the dynamics of the photoionization process (relative partial photoionization amplitudes including their phases and partial cross sections). The series of calculations sequentially investigates effects of the angular momentum coupling by initially assuming a pure coupling scheme for the atomic/ionic states (the geometrical model) and by later extending the approach to the intermediate-coupling approximation (a "generalized" geometrical model). In a particular case of the generalized geometrical model with the initial $L S J$-coupled state $\mathrm{Na}^{*} 2 p^{6} 3 p 2 P_{J_{0}}$, the final ionic state wave function is presented as a superposition of the $L S J$-coupled wave functions through $|\alpha J\rangle=$ $\sum_{L S} \gamma_{L S}^{\alpha J}|\alpha L S J\rangle$, where $\gamma_{L S}^{\alpha J}$ are mixing coefficients and $\alpha$ specifies the state. Neglecting mixing of configurations, term dependence of electron wave functions, as well as their photoelectron-energy dependence within the range of the $\mathrm{Na}^{+} 2 p^{5} 3 p$ configuration, the relative intensities of the photoelectron lines can be derived as $[1,8]$ :

$$
I_{\alpha J}=I_{0} \sum_{j=\frac{1}{2}, \frac{3}{2}}\left[\sum_{L S}(-1)^{\xi} \hat{j} \hat{J} \hat{L} \hat{S}\left\{\begin{array}{ccc}
1 & \frac{1}{2} & j \\
L & S & J \\
1 & \frac{1}{2} & J_{0}
\end{array}\right\} \gamma_{L S}^{\alpha J}\right]^{2},
$$

where $\xi=L+S+J$ and $I_{0}$ is a factor independent on $J$ and $J_{0}$. We have used conventional notation for the $9 j$ coefficient [14] and abbreviated $\hat{J}=\sqrt{2 J+1}$. Equa- tion (1) is a very particular case of a general equation for the relative intensities of lines in the angle-resolved photoelectron spectra from polarized targets [15]. We have separately calculated the values of $\gamma_{L S}^{\alpha J}$ for the $\mathrm{Na}^{+}$ $2 p^{5} 3 p$ configuration within the semirelativistic BreitPauli model with the Hartree-Fock electron wave functions [16]. Note that the relative intensities (1) do not imply any calculations of the photoionization amplitudes.

In the two lower panels in Fig. 1, we present the results of our calculations performed in the geometrical, i.e., $L S J$-coupling, model (shown as grey vertical bars) and the generalized geometrical model, Eq. (1) (shown as black vertical bars), for photoionization from the ${ }^{2} P_{3 / 2}$ [Fig. 1(c)] and the ${ }^{2} P_{1 / 2}$ [Fig. 1(d)]. The behavior of the ${ }^{3} D_{3},{ }^{3} D_{1},{ }^{3} P_{2}$, and ${ }^{3} P_{0}$ lines can be explained within the geometrical model [6] for ionization of a $p$ electron from a closed shell of an atom with the orbital angular momentum $L_{0}=1$, spin $S_{0}=\frac{1}{2}$, and total angular momentum $J_{0}$, into the ionic state with the corresponding quantum numbers $L S J$. Applying the $L S J$-coupling limit of Eq. (1) gives the ratios $(0: 1.75),(0.42: 1.04)$, and (1.25:0.125) for the intensities of the ${ }^{3} D_{3},{ }^{3} P_{2}$, and ${ }^{3} D_{1}$ lines in the ${ }^{2} P_{1 / 2}$ and ${ }^{2} P_{3 / 2}$ spectra, respectively. The predicted vanishing intensity of the line ${ }^{3} D_{3}$ in the ${ }^{2} P_{1 / 2}$ spectra is in accordance with the work of Pan and Starace [6]. In our case, a transition is quasiforbidden unless the relation $J \leq J_{0}+\frac{3}{2}$ is satisfied, which follows from the properties of the $9 j$ symbol in Eq. (1). In contrast, the experimental behavior of the four photolines, final ionic states ${ }^{3} D_{2},{ }^{1} D_{2},{ }^{1} P_{1}$, and ${ }^{3} P_{1}$, sharply contradicts the predictions of the geometrical model, as indicated in Fig. 1. The observed breakup of the geometrical model is explained by a violation of the $L S J$-coupling scheme in the final ionic states: the agreement between the generalized geometrical model (black vertical bars) and measured relative intensities seen in Fig. 1 is excellent. This directly shows that all assumptions of the generalized geometrical model are valid. Especially striking is an almost complete suppression of the line ${ }^{1} D_{2}$ in the ${ }^{2} P_{1 / 2}$ spectrum in contradiction with its geometrical intensity. The corresponding intermediatecoupled ionic wave function is of the form $\left|2 p^{5} 3 p^{1} D_{2}\right\rangle=$ $+0.793\left|{ }^{1} D\right\rangle+0.577\left|{ }^{3} P\right\rangle-0.197\left|{ }^{3} D\right\rangle$, i.e., the ${ }^{1} D$ component contributes only by approximately $63 \%$. The ${ }^{1} D_{2}$ line almost disappears in the ${ }^{2} P_{1 / 2}$ spectrum as a result of "negative interference" (i.e., a mutual cancellation) between product terms corresponding to different $L S$ components in Eq. (1). A further insight into this effect gives a decomposition of the $2 p^{5} 3 p^{1} D_{2}$ wave function in terms of the $j j$-coupled basis, which is the only other possible coupling scheme with fixed total angular momentum of the $3 p$ spectator electron. It results in $83 \%$, $13 \%$, and $4 \%$ contributions of the $2 p_{1 / 2}^{5} 3 p_{3 / 2}, 2 p_{3 / 2}^{5} 3 p_{3 / 2}$, and $2 p_{3 / 2}^{5} 3 p_{1 / 2}$ components, respectively. For direct photoionization into the $\mathrm{Na}^{+} 2 p^{5} 3 p^{1} D_{2}$ state from the $\mathrm{Na}^{*}$ 


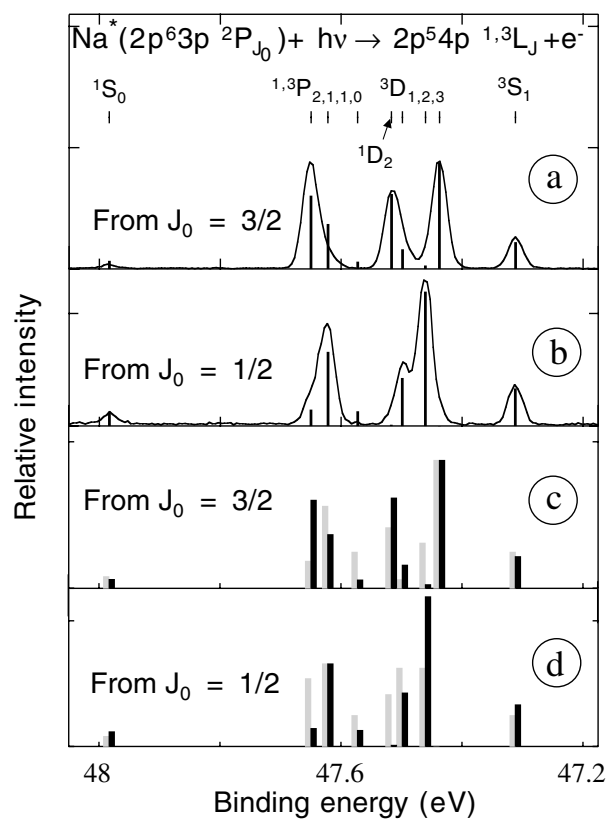

FIG. 2. Same as Fig. 1 but for the region of the $\mathrm{Na}^{+} 2 p^{5} 4 p$ shakeup photoelectron spectra.

$2 p^{6} 3 p^{2} P_{1 / 2}$ fine-structure state, only the last weak component contributes. This example shows that some fine-structure lines can "accidentally" disappear in photoelectron spectra: an effect which is independent of the photon energy and which one cannot predict only using selection rules based on pure coupling schemes. It is clearly seen here without any ambiguity. One can call such lines "dynamically forbidden," since dynamical atomic structure calculations are needed to find that the corresponding cross section vanishes. The numerical HF results presented in Figs. 1(a) and 1(b) employ the intermediate-coupling ionic wave functions and therefore automatically include the above cancellation effect.

Our observations on the main $2 p^{5} 3 p$ photoelectron lines are fully confirmed by the measurements of the shakeup satellites $2 p^{5} 4 p$ shown together with the results of the HF calculations in the two upper panels in Fig. 2 as excited from the $\mathrm{Na}^{*} 2 p^{6} 3 p^{2} P_{3 / 2}$ [Fig. 2(a)] and ${ }^{2} P_{1 / 2}$ [Fig. 2(b)] states. Assuming the pure shakeup excitation mechanism of the $\mathrm{Na}^{+} 2 p^{5} 4 p$ states, the geometric relative line intensities remain as for the $\mathrm{Na}^{+} 2 p^{5} 3 p$ states (grey vertical bars in the two lower panels), while for the intermediate coupling the set of $\gamma$ coefficients in (1) has been calculated specifically for the $2 p^{5} 4 p$ configuration. The theoretical relative intensities in the generalized geometrical model (dark vertical bars in the lower panels in Fig. 2) are again in very good agreement with the measurements, indicating that the same approach is also valid for the shakeup satellites.
In conclusion, we have performed the first very highresolution photoelectron experiments for photoionization of open-shell atoms in which the total angular momentum $J_{0}$ could be changed by laser excitation of the initial state and the resulting photolines individually resolved. We observed dramatic variations in the relative intensities of the photolines. Some of these are explained in terms of quasiforbidden transitions and corresponding selection rules as understood in a geometrical formulation of the photoionization process. Additional dynamically forbidden transitions were discovered. The corresponding lines are almost completely suppressed despite the lack of explicit selection rules. They arise due to a mutual cancellation of terms in the photoionization cross section originating from different components of the final-ion wave functions.

This work was supported in part by the Chemical Sciences, Geosciences and Biosciences Division, Office of Basic Sciences, Office of Science, DoE, and by the Scientific Affair Division of NATO under Contract No. PST-CLG 980080. The authors thank the ALS and Enterprise Ireland for helpful support.

[1] J. Berkowitz and G. L. Goodman, J. Chem. Phys. 71, 1754 (1979); G. L. Goodman and J. Berkowitz, ibid. 94, 321 (1991).

[2] M. O. Krause et al., Phys. Rev. A 47, 3015 (1993).

[3] S. B. Whitfield et al., Phys. Rev. Lett. 84, 4818 (2000).

[4] Ph. Wernet et al., Phys. Rev. A 64, 042707 (2001), and references therein.

[5] A. Dorn et al., J. Phys. B 28, L225 (1995); A. N. GrumGrzhimailo and A. Dorn, J. Phys. B 28, 3197 (1995).

[6] C. Pan and A. F. Starace, Phys. Rev. A 47, 295 (1993).

[7] N. Beatham et al., Chem. Phys. Lett. 63, 69 (1979).

[8] J. Schirmer et al., Phys. Rev. A 22, 2696 (1980).

[9] For overviews of earlier results, see B. Sonntag and P. Zimmermann, Rep. Prog. Phys. 55, 911 (1992); M. O. Krause and C. D. Caldwell, in VUV and Soft X-Ray Photoionization, edited by U. Becker and D. A. Shirley (Plenum, New York, 1996), p. 181; J. Schulz et al., Phys. Rev. A 67, 012502 (2003), and references therein.

[10] N. Berrah et al., J. Electron Spectrosc. Relat. Phenom. 101-103, 1 (1999).

[11] D. Cubaynes et al., Phys. Rev. Lett. 63, 2460 (1989).

[12] M. Meyer et al., Phys. Scr., T 31, 28 (1990).

[13] R. D. Cowan, The Theory of Atomic Structure and Spectra (University of California, Berkeley, 1981).

[14] D. A. Varshalovich, A. N. Moskalev, and V. K. Khersonskii, Quantum Theory of Angular Momentum (World Scientific, Singapore, 1988).

[15] A. N. Grum-Grzhimailo (private communication).

[16] C. Froese Fischer et al., Computational Atomic Structure. An MCHF Approach (Institute of Physics, Bristol, 1997). 